\title{
AN OPTIMIZATION APPROACH TO THE CHOICE OF PARAMETERS OF LINEAR PULSE INDUCTION ELECTROMECHANICAL CONVERTER
}

Purpose. The purpose of the paper is to select the main parameters of the linear pulse induction electromechanical converters (LPIEC) for high-speed and power use with the use of the optimization approach, which provides an increase in speed and power indicators with limited electric, thermal and mass-dimensions. Methodology. A technique for finding the maximum of the integral efficiency criterion of LPIEC in the search space using a global optimization method that randomly searches for parameters, preventing entry into a local maximum, and a local method ensuring the contraction of the range of parameters with a global maximum to minimum dimensions is developed. As a global optimization method, genetic algorithms are used, and the Nelder-Mead method is used as the local method. Results. The LPIEC inductor should have a maximum external and minimum internal diameter, and its height should be less than that of the LPIEC of the basic design. The armature should have a maximum outer diameter, and the thickness of its wire should be minimal. The armature should be made with a significantly higher height, a greater number of turns and a wider wire. The height of the LPIEC inductor for power purposes should be almost the same as that of the LPIEC of the basic design. In this case, the number of turns of the inductor and the cross section of its wire should be approximately the same. The armature should be made with a slightly larger inner diameter and a significantly higher height. This armature should have a larger number of turns of wire, which must be stacked in 4 layers, and a large width of the wire. The average energy value and voltage of the capacitive energy storage for the LPIEC for high-speed and power applications should be higher than for the LPIEC of the basic design. Originality. An optimization approach to the choice of LPIEC parameters with a multi-turn squirrel arm is developed, which consists in finding the maximum of an integral efficiency criterion that takes into account the maximum speed and efficiency in a high-speed converter, the amplitude and magnitude of the electrodynamic force pulse in a power converter, with minimum temperature excesses, the mass of active elements and current of the inductor. The optimization uses a chain mathematical model that takes into account the interconnected electrical, magnetic, thermal and mechanical processes of the LPIEC. Practical value. The electric parameters of the capacitive energy storage device and the geometric parameters of the LPIEC are determined, which ensure the largest values of the integral efficiency criterion depending on the adopted version of the efficiency evaluation strategy. In optimized speed and power transfer converters, the integral efficiency criteria are 2.2 times higher on average than in the basic performance of the LPIEC. References 14, tables 6, figures 2.

Key words: linear pulse induction electromechanical converter, chain mathematical model, integral efficiency criterion, optimization approach, genetic algorithms, Nelder-Mead method.

Разработан оптимизационный подход к выбору параметров линейного импульсного индукционного электромеханического преобразователя (ЛИИЭП) с многовитковым короткозамкнутым якорем. Он состоит в нахождении максимума интегрального критерия эффективности, учитывающего максимальную скорость и КПД преобразователя скоростного назначения, амплитуду и величину импульса электродинамических усилий в преобразователе силового назначения при минимальных превышениях температур, массе активных элементов и токе индуктора. При этом используется цепная математическая модель, которая учитывает взаимосвязанные электрические, магнитные, тепловые и механические процессы ЛИИЭП. Разработана методика поиска максимума интегрального критерия эффективности ЛИИЭП в поисковом пространстве с использованием глобального и локального методов оптимизации. В качестве глобального метода используются генетические алгоритмы, а в качестве локального - метод Нелдера-Мида. Установлены электрические параметры емкостного накопителя энергии и геометрические параметры ЛИИЭП, обеспечивающие наибольшие значения интегрального критерия эффективности в зависимости от принятого варианта стратегии оценки эффективности. В оптимизированных преобразователях скоростного и силового назначения интегральные критерии эффективности в среднем в 2,2 раза выше, чем в ЛИИЭП основного исполнения. Библ. 14, табл. 6, рис. 2.

Ключевые слова: линейный импульсный индукционный электромеханический преобразователь, цепная математическая модель, интегральный критерий эффективности, оптимизационный подход, генетические алгоритмы, метод Нелдера-Мида.

Introduction. Linear pulse electromechanical converters are widely used to accelerate the actuator (A) to high speed in a short active area and/or to create powerful power pulses on the object of action with a slight movement of A, made, for example, as a striker [1-4]. Such converters of high-speed and power purposes are used in many branches of science and technology as electromechanical accelerators and shockpower devices [5].

The most widely used converters are of induction type, which have a coaxial disk configuration. Such linear pulsed induction electromechanical converters (LPIEC) contain an accelerated electrically conductive armature that magnetically interacts with a fixed inductor [6-8]. When the inductor is excited, a current is induced from a capacitive energy storage (CES) in an electrically conductive armature. The interaction of the inductor's magnetic field with induced current leads to the occurrence of electrodynamic forces (EDF), causing axial displacement of the armature with $\mathrm{A}$. In this case, it is considered expedient to excite the inductor by a

(C) V.F. Bolyukh, I.S. Schukin 
polar aperiodic pulse, which allows the use of electrolytic capacitors with an increased specific energy index for the CES [5].

However, at operation with a rapid change in the electromagnetic, mechanical and thermal parameters, the efficiency of the power and speed indicators of LPIEC is not high enough. One of the ways to improve these indicators is to use an optimization approach to the choice of the main parameters of LPIEC. With this choice, it is advisable to use the integral efficiency criterion, which should include the main speed or power indicators of LPIEC, taking into account its electrical, thermal and mass-dimensional indicators.

The goal of the paper is the choice of the basic parameters of LPIEC for speed and power purposes using an optimization approach that provides an increase in speed and power indicators with limited electrical, thermal and mass-dimensional indicators.

To increase the speed of the computational algorithm we use the chain mathematical model of LIIEP, which uses lumped parameters of the inductor and armature [9, 10]. This model takes into account interconnected electrical, magnetic, thermal and mechanical processes. To eliminate the influence of the skin effect, we consider an armature made as a shortcircuited multi-turn winding tightly wound with a relatively thin copper wire.

Parameters and indicators of LPIEC. The optimization process consists in finding a set of parameters that provide the maximum values of velocity and kinetic energy in LPIEC for high-speed assignment and maximum values of amplitude and value of the impulse of EDF in LPIEC for power purposes. These indicators should be provided with the minimum temperature increases and mass of the $n$-th active elements $(n=1,2$ are the indices of the inductor and armature, respectively) and the minimum current of the inductor, which is important for the control system.

\section{Main LPIEC parameters}

CES electrical parameters:

- $U_{0}, W_{0}-$ CES voltage and energy, respectively. LPIEC geometrical parameters for the $n$-th active element:

- $w_{n}$ - number of turns of the wire;

- $d_{0 n}$ - diameter of round wire;

- $h_{z n}, h_{r n}$ - height and width of rectangular wire;

- $D_{\text {ex } n}, D_{\text {in } n}-$ outer and inner diameters;

- $H_{n}$ - axial height. LPIEC additional parameters:

- $m_{2}, m_{e}$ - mass of the armature and A, respectively;

- $\Delta z_{0}=0.5 \cdot\left(H_{1}+H_{2}\right)+\Delta_{0} \quad-\quad$ initial axial displacement between the centers of the $n$-th active elements, where $\Delta_{0}$ - initial gap between active elements;

- converter shape: disk or cylindrical feedthrough (armature inside the inductor or vice versa);

- type of armature (multiturn, massive, combined);

- circuit of the formation of a current pulse in the inductor when excited from the CES;
- initial temperature $T_{0 n}$ of the $n$-th active element;

- mechanical factors: the forces of resistance to the movement of the armature, friction, etc.;

- parameters of lead wires and connecting elements.

These parameters are subject to parametric and functional limitations:

for energy source

$W_{0 \min } \leq 0.5 \cdot C \cdot U_{0}^{2} \leq W_{0 \max }-$ on CES energy,

where $C=2 W_{0} U_{0}^{-2}-$ CES capacitance;

for load

$0 \leq m_{e} \leq m_{e \max }-$ on mass of the accelerated A;

$f_{c \text { min }} \leq f_{c} \leq f_{c \max }$ - on braking and opposing forces; for electronic control system

$i_{1} \leq i_{1 \max }-$ limitation on the amplitude of the inductor excitation current;

for geometrical parameters

$1 \leq w_{n} \leq \operatorname{Ent}\left(0.5 \frac{D_{e x n}-D_{i n n}}{h_{r n}+2 h_{s}}\right) \cdot \operatorname{Ent}\left(\frac{H_{n}}{h_{z n}+2 h_{s}}\right)-$ for the number of turns of rectangular wire, where $\operatorname{Ent}(f)$ - the largest integer not exceeding $f ; h_{s}$ - winding conductor insulation thickness;

$1 \leq \operatorname{Ent}\left(0.5 \frac{D_{e x n}-D_{i n n}}{h_{r n}+2 h_{s}}\right) \leq K_{w n \max }$ - for the number of layers of rectangular wire;

$0 \leq \Delta z_{0} \leq 0.5 \cdot\left(H_{1}+H_{2}\right) ; \quad 0 \leq D_{\text {in } 2} \leq D_{\text {in } 2 \max } ;$

$D_{i n 2}+2 \cdot\left(h_{r 2}+2 \cdot h_{s}\right) \leq D_{e x 2} \leq D_{i n 1}-\Delta_{0}-$ for cylindrical feedthrough converter, there $D_{\text {in } 2 \max }-$ maximum value of the armature inner diameter;

When using a round wire in parametric constraints, instead of $h_{r n}$ and $h_{z n}$ it is necessary to use its diameter $d_{0 n}$; $i_{1}(t) i_{2}(t) \frac{d M_{12}}{d z}(z) \leq f_{z \max }$ - on the amplitude of EDF active along the $z$-axis; where $i_{n}(t)$ - current of the $n$-th active element, $M_{12}-$ mutual inductance between active elements; $F_{z \min } \geq \int_{0}^{t_{p}} f_{z} d t \geq F_{z \max }-$ on the EDF impulse value, where $t_{p}$ is the duration of the EDF action; $W_{k i n \min } \geq 0.5 \cdot\left(m_{2}+m_{e}\right) V_{p}^{2} \geq W_{k i n \max }$ - on the kinetic energy, where $V_{p}$ is the armature velocity at the end of the operation process; $V_{\min } \geq v \geq V_{\max }$ - on the velocity of displacement of the armature with $\mathrm{A} ; \theta_{n} \leq \theta_{n \max }-$ on the maximum permissible temperature rise of the $n$-th active element.

LPIEC of basic design. As the basic design, we consider LPIEC with following parameters: [11]:

Inductor: outer diameter $D_{e x 1}=100 \mathrm{~mm}$, inner diameter $D_{i n 1}=10 \mathrm{~mm}$, height $H_{1}=10 \mathrm{~mm}$. The inductor is made as a two-layer winding with external electrical leads; rectangular wire cross section $h_{z 1} \times h_{r l}=1.8 \times 4.8 \mathrm{~mm}^{2}$, number of turns of the wire $w_{1}=46$. 
Armature: outer diameter $D_{e x 2}=100 \mathrm{~mm}$, inner diameter $D_{i n 2}=6 \mathrm{~mm}$, height $H_{2}=2.5 \mathrm{~mm}$. The armature is made as a multilayer short-circuited winding, the cross section of a rectangular copper wire $h_{z 2} \times h_{r 2}=1.0 \times 1.2 \mathrm{~mm}^{2}$, number of turns of the wire $w_{2}=80$.

CES: capacitance $C_{0}=3 \mathrm{mF}$, voltage $U_{0}=0.4 \mathrm{kV}$.

Initial distance between inductor and armature $\Delta_{0}=1 \mathrm{~mm}$.

In LPIEC for speed purposes, the return spring's coefficient of elasticity is $K_{P}=50 \mathrm{kN} / \mathrm{m}$. Mass of A $m_{e}=0.5 \mathrm{~kg}$. We believe that in LPIEC of power designation the counteracting force is significant and there is no movement of A.

In LPIEC of the basic design of speed purpose, the following indicators are implemented: the amplitude of the inductor current $i_{1 m}=2.57 \mathrm{kA}$, the maximum current density in the inductor's conductor $j_{1 m}=297.5 \mathrm{~A} \mathrm{~mm}^{2}$, the maximum current density in the armature $j_{2 m}=764.56 \mathrm{~A} / \mathrm{mm}^{2}$, the amplitude of EDF $f_{z m}=13.983 \mathrm{kN}$, the value of the impulse of EDF $F_{z}=5.674 \mathrm{Ns}$, the maximum speed of the armature with the inductor $V_{m}=8.43 \mathrm{~m} / \mathrm{s}$, the efficiency $\eta=10.32 \%$, the temperature rise of the inductor $\theta_{1}=0.37{ }^{\circ} \mathrm{C}$, the temperature rise of the armature $\theta_{2}=0.97^{\circ} \mathrm{C}$. The mass of copper in the inductor is $m_{1}=0.69 \mathrm{~kg}$, the mass of copper in the armature is $m_{2}=0.17 \mathrm{~kg}$.

The following indicators are implemented in the LPIEC of the basic design of power purpose: the amplitude of the inductor current $i_{1 m}=2.953 \mathrm{kA}$, the maximum current density in the inductor's conductor $j_{1 m}=341.78 \mathrm{~A} / \mathrm{mm}^{2}$, the maximum current density in the armature $j_{2 m}=893.51 \mathrm{~A} / \mathrm{mm}^{2}$, the amplitude of EDF $f_{z m}=20.171 \mathrm{kN}$, the magnitude of the impulse of EDF $F_{z}=9.076 \mathrm{Ns}$, the temperature rise of the inductor $\theta_{1}=0.4{ }^{\circ} \mathrm{C}$, the temperature rise of the armature $\theta_{2}=1.45^{\circ} \mathrm{C}$.

Integral efficiency criterion. Since the efficiency of LPIEC of speed or power purpose is characterized by a number of versatile indicators, we introduce an integral efficiency criterion, which takes into account the maximum speed or power indicators with minimum values of the inductor current amplitude, temperature rises and the total mass of copper wire of active elements. In a dimensionless form, it can be written as follows.

$$
K^{*}=\frac{\beta_{1}}{i_{1 m}^{*}}+\beta_{2} A^{*}+\beta_{3} B^{*}+\frac{\beta_{4}}{\theta_{1}^{*}}+\frac{\beta_{5}}{\theta_{2}^{*}}+\frac{\beta_{6}}{m_{\Sigma}^{*}} ; \sum_{j=1}^{J} \beta_{j}=1,
$$

where $\beta_{j}$ are the weights of the corresponding indicator; $J=6$ is the number of functional indicators, normalized relative to LPIEC of the basic design (marked with asterisks);

$m_{\Sigma}=0.5 \pi \gamma_{C u} \sum_{n=1}^{2}\left(D_{\text {exn }}+D_{i n n}\right) h_{r n} h_{z n} w_{n}$ is the total mass

of the copper wire in the $n$-th active elements,

where $\gamma_{C u}$ is the copper wire's density;

$A=f_{z m}, B=F_{z}$ - for LPIEC of power purpose;

$A=V_{z m}, B=\eta-$ for LPIEC of speed purpose;

$f_{z m}$ is the EDF amplitude;

$V_{z m}$ is the maximum speed of the armature with A; $\eta=100 \frac{\left(m_{2}+m_{e}\right) v_{z}^{2}+K_{P} \Delta z^{2}}{C_{0} U_{0}^{2}}, \% \quad$ is the LPIEC efficiency.

Note that LPIEC of a basic design of speed or power designation has $K^{*}=1$. The best will be the converter with the maximum value of $K^{*}$ showing how many times it is more efficient than LPIEC of the basic design.

A technique of finding the maximum of the target function. The integral efficiency criterion of LPIEC $K^{*}$ is a target function of the optimization process. The strategy for finding the maximum of the target function of $m$ variables in the search space is to share the global optimization method that performs a random search for LPIEC parameters in a given space, preventing it from falling into a local extremum, and a local method that provides tightening the parameter area with a global extremum to the minimum sizes.

As a global optimization method, we use genetic algorithms based on the mechanisms of population genetics $[12,13]$. According to this method, each attribute of an object in the phenotype corresponds to one gene in the genotype, which is a bit string of fixed length. The sign is divided into tetrads, converted by the Gray code. When encoding a binary string of $\iota$ bits of the variable $x_{k}$, which belongs to the segment $\left[x_{\min }, x_{\max }\right]$, each string $s_{k}$ expresses the value of the variable $x_{k}$ :

$$
x_{k}=x_{\min }+s_{k}\left(x_{\max }-x_{\min }\right) / 2^{l},
$$

where $s_{k}$ is the value of the binary number encoded by this string.

Operating on a set (population) of possible solutions $P=\left(x_{1}, \ldots, x_{m}\right)$, the set of parameters $x_{i}$, of structured in a certain way in the form of a chain of finite length is processed, and subsequent generations of the solution population are generated using genetic operators. Thus, a randomized search with centralized control is implemented, using selection and genetic mechanisms of reproduction, with an arbitrary choice of points of application of operators.

Genetic algorithms can be represented as follows:

$$
\mathrm{GA}=\left(\boldsymbol{P}^{0}, m, l, S, Q, l, \xi\right),
$$

where $\boldsymbol{P}^{0}=\left(a_{1}^{0}, \ldots, a_{m}^{0}\right)$ is the initial population; $a_{i}^{0}$ is the problem solution in the form of a chromosome, $i=\overline{1, m} ; m$ is the population dimension: $l$ is the length of each chromosome of the population; $S$ is the selection operator; $Q$ is the recombination mapping recombination (crossover, mutation); $\imath$ is the optimality function; $\xi$ is the break criterion.

The work of genetic algorithms is an iterative process that continues until a given condition is met, for example, slowing down the growth of the efficiency criterion $K^{*}=1$ to a given value. $\boldsymbol{P}^{0}$ is a randomly generated initial population. At each iteration cycle, selection, crossover, and mutation operators are implemented. The selection operator $S$ generates an intermediate population $\boldsymbol{R}^{t}$ from the population $\boldsymbol{P}^{t}$ by 
selecting and generating new copies of the elements $\boldsymbol{P}^{t}$ : $\boldsymbol{R}^{t}=S\left(\boldsymbol{P}^{t}\right)$. The optimality function $l$, which provides feedback on the results of optimization during generation $t$, is used to select individuals in the population. The selection is made based on the probabilities $p_{S}\left(a_{i}^{t}\right)$ calculated for each individual:

$$
p_{S}\left(a_{i}^{t}\right)=\frac{\imath\left(a_{i}^{t}\right)}{\sum_{j=1}^{m} l\left(a_{j}^{t}\right)} .
$$

After the selection is completed, for the element $a_{i}^{t} \in \boldsymbol{R}^{t}$ a partner is selected from $\boldsymbol{R}^{t}$ for recombination and a new chromosome is built.

The crossover with probability $p_{C}$ is performed as follows:

- random selection of crossbreeding partners

$$
a_{1}=\left(a_{1,1} \ldots a_{1, l}\right) \in \boldsymbol{R}^{t}, \quad a_{2}=\left(a_{2,1} \ldots a_{2, l}\right) \in \boldsymbol{R}^{t}
$$

- random crossover point selection $x \in\{1, \ldots, l-1\}$;

- formation of two new individuals

$$
\begin{aligned}
& a_{1}^{\prime}=\left(a_{1,1} \ldots a_{1, x} a_{2, x+1} \ldots a_{2, l}\right), \\
& a_{2}^{\prime}=\left(a_{2,1} \ldots a_{2, x} a_{1, x+1} \ldots a_{1, l}\right) \text {. }
\end{aligned}
$$
bit:

A mutation is a random change in the chromosome

- random selection with probability $p_{M}$ of positions $\left\{x_{1}, \ldots, x_{k}\right\} \subseteq\{1, \ldots, l\} \quad$ inside the bit string $a=\left(a_{1} \ldots a_{l}\right) \in \boldsymbol{R}^{t}$, prone to mutation;

- formation of a new individual $a=\left(a_{1} \ldots a_{x_{1}-1} \overline{a_{x_{1}}} a_{x_{1}+1} \ldots a_{x_{i}-1} \overline{a_{x_{i}}} a_{x_{i}+1} \ldots a_{l}\right),(i=\overline{1, k})$.

As a local optimization method for finding the maximum of the optimality criterion $K^{*}(X)$ in the $n$ dimensional Euclidean space $R^{n}$

$$
\max K^{*}(X)=K^{*}\left(X^{*}\right), \quad X \in R^{n}
$$

the Nelder-Mead method is used changing the current simplex [14].

As a result of reflection of the $k$-th vertex of the simplex with the coordinates of the vertices $X_{i}^{r}, i \in[1, n+1]$, a simplex is formed with the coordinates of the vertices

$$
X_{i}^{r+1}=X_{i}^{r}, i \in[1, n+1], i \neq k, X_{k}^{r+1}=2 X_{C}^{r}-X_{k}^{r},
$$

where $X_{C}^{r}=\frac{1}{n} \sum_{i=1, i \neq k}^{n+1} X_{i}^{r}$ is the vector of coordinates of the center of gravity of the remaining vertices of the simplex.

As a result of the reduction of the vertices of the simplex $X_{i}^{r}$ to the vertex $X_{k}$, we obtain the simplex with the coordinates of the vertices

$$
X_{i}^{r+1}=X_{k}^{r}+\gamma\left(X_{i}^{r}-X_{i}^{k}\right), i \in[1, n+1], i \neq k, X_{k}^{r+1}=X_{k}^{r},
$$

where $\gamma \in(0.1), \gamma \approx 0.5$ is the reduction factor.
After the operation of compressing the simplex $X_{i}^{r}$ in the direction $\left(X_{k}^{r}-X_{C}^{r}\right)$, we obtain a simplex with the coordinates of the vertices $X_{i}^{r+1}=X_{k}^{r}, i \in[1, n+1], i \neq k, X_{k}^{r+1}=X_{C}^{r}+\beta\left(X_{k}^{r}-X_{C}^{r}\right)$, where $\beta \in(0.1), \beta \approx 0.4-0.6$ is the compression factor.

As a result of extension of the simplex $X_{i}^{r}$ in the direction $\left(X_{k}^{r}-X_{C}^{r}\right)$, we obtain a simplex with the coordinates of the vertices

$$
X_{i}^{r+1}=X_{i}^{r}, i \in[1, n+1], i \neq k, X_{k}^{r+1}=X_{C}^{r}+\alpha\left(X_{k}^{r}-X_{C}^{r}\right),
$$

where $\alpha \approx 2.8-3.0$ is the extension factor.

Since the deformation procedure is repeated many times, the polyhedron adapts to the local relief of the target function and shrinks, ensuring the convergence of the algorithm in the local maximum, allowing by the size of the polyhedron $\sigma_{i}$ to judge the stage of the search for the converter parameters.

Realization of the task of choosing the parameters of LPIEC. Consider a LPIEC of a disk configuration with a multiturn armature that is excited by a polar aperiodic pulse (CES is shunted by a reverse diode). The following are used as independent variables that are included in the LPIEC design variables vector: outer $D_{e x n}$ and inner $D_{i n n}$ diameters, height $H_{n}$, number of turns $w_{n}$, height $h_{z n}$, and width $h_{r n}$ of a rectangular wire of the $n$-th active element; voltage $U_{0}$ and energy $W_{0}$ of CES. Restrictions on these parameters impose the boundaries of the search space (Table 1).

Table 1

Functional and parametric limitations of LPIEC parameters

\begin{tabular}{|l|c|}
\hline \multicolumn{1}{|c|}{ Parameter } & Value \\
\hline CES energy $W_{0}, \mathrm{~J}$ & $150 \ldots 500$ \\
\hline CES voltage $U_{0}, \mathrm{~V}$ & $150 \ldots 500$ \\
\hline Outer diameter of the $n$-th active elements $D_{e x n}, \mathrm{~mm}$ & $50 \ldots 100$ \\
\hline Inner diameter of the inductor $D_{i n 1}, \mathrm{~mm}$ & $10 \ldots 20$ \\
\hline Inner diameter of the armature $D_{i n 2}, \mathrm{~mm}$ & $2 \ldots 20$ \\
\hline Inductor height $H_{1}, \mathrm{~mm}$ & $5 \ldots 22$ \\
\hline Armature height $H_{2}, \mathrm{~mm}$ & $1 \ldots 10$ \\
\hline Number of inductor layers $K_{w 1}$ & 2 \\
\hline Number of turns of inductor $w_{1}$ & $30 \ldots 75$ \\
\hline Inductor wire height $h_{z 1}, \mathrm{~mm}$ & $1 \ldots 2$ \\
\hline Inductor wire width $h_{r 1}, \mathrm{~mm}$ & $2 \ldots 10$ \\
\hline Number of armature layers $K_{w 2}$ & $1 \ldots 8$ \\
\hline Number of turns of armature $w_{2}$ & $20 \ldots 200$ \\
\hline Armature wire height $h_{z 2}, \mathrm{~mm}$ & $0.5 \ldots 1.5$ \\
\hline Armature wire width $h_{r 2}, \mathrm{~mm}$ & $1.0 \ldots 3.0$ \\
\hline Wire insulation thickness $h_{s}, \mathrm{~mm}$ & 0.1 \\
\hline Initial gap between the $n$-th active elements $\Delta_{0}, \mathrm{~mm}$ & 1.0 \\
\hline
\end{tabular}

For optimization calculations, a computational algorithm was applied, which includes the following steps [7].

1. A genetic representation of the polyhedron is specified by a set of $N+1$ parameters - vectors of design variables $P^{0}=\left(\boldsymbol{x}_{1}^{0}, \ldots, \boldsymbol{x}_{N+1}^{0}\right), \boldsymbol{x}\left(x_{1}, \ldots, x_{N}\right) \in \mathfrak{R}^{N}$. 
2. From $K$ source polyhedra $P_{i}^{0}=\left(\boldsymbol{x}_{i, 1}^{0}, \ldots, \boldsymbol{x}_{i, N+1}^{0}\right)$, $i=1, \ldots, K$ the population $D_{i}\left(P_{i}^{0}\right)$ is randomly formed.

3. The operators of reflection, extension, compression, and reduction are applied to each polyhedron $P_{i}^{0}$ to perform a specified number of steps $s$ in the search space.

4. The value of the target function $F^{t}\left(x_{i, j}^{t}\right), i=1, . . K, j=1, . . N+1$ is determined in each vertex of the polyhedron as well as its the «best» vertex $\boldsymbol{x}_{i, b}^{t}, i=1, . . K$.

5. The polyhedra are ranked relative to the value of the target function of their best vertexes. $F_{b}^{t}\left(\boldsymbol{x}_{i, b}^{t}\right), i=1, . . K$.

6 . The polyhedron with the worst parameters is eliminated.

7. A new polyhedron $P_{K}^{t}$ is formed by applying genetic operators of the crossover and mutation, acting with probability $\rho_{\text {mut }}$, to two randomly selected polyhedra from the remaining $(K-1)$.

8. The value of the target function $F\left(\boldsymbol{x}_{K, j}^{t}\right), j=1, . . N+1$ and the «best» vertex of the polyhedron $P_{K}^{t}$ are determined.

9. Polyhedrons $P_{i}^{t}$ are ranked by size $\sigma\left(P_{i}^{t}\right), i=1, . . K$.

10. Threshold $\sigma^{t}$ is determined for getting into the search group by size of the $h$-th population $\sigma\left(P_{h}^{t}\right)$.

11. To $(K-h)$ populations operators of reflection, extension, compression and reduction are applied.

12. Return to step 4.

The results of the choice of parameters of LPIEC of speed purpose. The choice of parameters is largely determined by the adopted version of the strategy for assessing the effectiveness of LPIEC. Consider four options for the strategy (Table 2).

Table 2

The weights of the options for the evaluation strategy for the LPIEC efficiency, p.u.

\begin{tabular}{|c|c|c|c|c|c|c|}
\hline Strategy options & $\beta_{1}$ & $\beta_{2}$ & $\beta_{3}$ & $\beta_{4}$ & $\beta_{5}$ & $\beta_{6}$ \\
\hline I & 0.2 & 0.2 & 0.2 & 0.1 & 0.1 & 0.2 \\
\hline II & 0.1 & $\mathbf{0 . 3}$ & $\mathbf{0 . 3}$ & 0.1 & 0.1 & 0.1 \\
\hline III & 0.05 & $\mathbf{0 . 5}$ & 0.2 & 0.1 & 0.1 & 0.05 \\
\hline IV & 0.05 & 0.2 & $\mathbf{0 . 5}$ & 0.1 & 0.1 & 0.05 \\
\hline
\end{tabular}

In option I, all LPIEC indicators are estimated equally (the total value of the temperature rise indicator is 0.2 ). In option II, the maximum speed $V_{m}$ and the efficiency $\eta$ of the converter are estimated to be the highest and equivalent. In option III, the maximum speed $V_{m}$ is most highly estimated, and in option IV efficiency $\eta$.

As a result of calculations for each of the options of the strategy for evaluating the effectiveness, the relative parameters of LPIEC for speed purpose were obtained: energy $W_{0}{ }^{*}$, voltage $U_{0}{ }^{*}$, and capacitance $C^{*}$ of CES; outer $D_{e x 1}$ and inner $D_{i n 1}{ }^{*}$ diameters, height $H_{1}{ }^{*}$, number of turns $w_{1}{ }^{*}$, thickness $h_{z 1}{ }^{*}$ and width $h_{r 1}{ }^{*}$ of the wire of the inductor; outer $D_{e x 2}{ }^{*}$ and inner $D_{i n 2}{ }^{*}$ diameters, height $H_{2}{ }^{*}$, number of turns $w_{2}{ }^{*}$ and number of layers $K_{w 2}{ }^{*}$, thickness $h_{z 2}{ }^{*}$ and width $h_{r 2}{ }^{*}$ of the wire of the armature (Table 3).

Table 3

Relative parameters of LPIEC of speed purpose

\begin{tabular}{|c|c|c|c|c|c|}
\hline \multirow{2}{*}{ Parameter } & \multicolumn{4}{|c|}{$\begin{array}{l}\text { Strategy options for estimation of } \\
\text { LPIEC efficiency }\end{array}$} & \multirow{2}{*}{$\begin{array}{c}\text { Average } \\
\text { value }\end{array}$} \\
\hline & I & II & III & IV & \\
\hline$D_{e x 1}{ }^{*}$ & 1.0 & 1.0 & 1.0 & 1.0 & 1.0 \\
\hline$D_{i n 1}{ }^{*}$ & 1.1 & 1.0 & 1.0 & 1.0 & 1.0 \\
\hline$H_{1}{ }^{*}$ & 0.62 & 0.62 & 0.82 & 0.66 & 0.68 \\
\hline$w_{1}{ }^{*}$ & 1.63 & 1.39 & 0.87 & 0.95 & 1.21 \\
\hline$h_{z 1}{ }^{*}$ & 0.55 & 0.67 & 1.11 & 1.11 & 0.86 \\
\hline$h_{r 1}{ }^{*}$ & 0.625 & 0.625 & 0.830 & 0.662 & 0.686 \\
\hline$D_{e x 2^{*}}{ }^{*}$ & 1.0 & 1.0 & 1.0 & 1.0 & 1.0 \\
\hline$D_{i n 2}{ }^{*}$ & 1.2 & 1.3 & 1.1 & 1.0 & 1.15 \\
\hline $\mathrm{H}_{2}{ }^{*}$ & 4.8 & 6.8 & 1.6 & 2.52 & 3.93 \\
\hline$w_{2}^{*}$ & 2.2 & 2.1 & 1.2 & 1.5 & 1.75 \\
\hline$K_{w 2}{ }^{*}$ & 2.0 & 2.0 & 1.0 & 1.5 & 1.63 \\
\hline$h_{z 2}{ }^{*}$ & 1.0 & 1.0 & 1.0 & 1.0 & 1.0 \\
\hline$h_{r 2}{ }^{*}$ & 2.08 & 3.33 & 1.5 & 1.66 & 2.14 \\
\hline
\end{tabular}

The operation of LPIEC is estimated by the following relative indicators: inductor current amplitude $i_{1 m}{ }^{*}$, maximum current density in conductors of inductor $j_{1 m}{ }^{*}$ and armature $j_{2 m}{ }^{*}$, maximum speed $V_{m}{ }^{*}$ and efficiency $\eta^{*}$, temperature rise of the inductor $\theta_{1}{ }^{*}$ and armature $\theta_{2}{ }^{*}$ at the end of the working process, total mass of the wire $m_{\Sigma}{ }^{*}$ and the criterion of efficiency $K^{*}$ (Table 4$)$.

Table 3, 4 also show the average values of the parameters and indicators of LPIEC for speed purpose.

Table 4

Relative indicators of LPIEC of speed purpose

\begin{tabular}{|c|c|c|c|c|c|}
\hline \multirow[t]{2}{*}{ Indicator } & \multicolumn{4}{|c|}{$\begin{array}{l}\text { Strategy options for estimation of } \\
\text { LPIEC efficiency }\end{array}$} & \multirow{2}{*}{$\begin{array}{c}\text { Average } \\
\text { value }\end{array}$} \\
\hline & I & II & III & IV & \\
\hline$W_{0}^{*}$ & 0.729 & 0.937 & 2.08 & 1.458 & 1.301 \\
\hline$U_{0}{ }^{*}$ & 0.437 & 0.562 & 1.25 & 1.25 & 0.875 \\
\hline$C^{*}$ & 3.8 & 2.96 & 1.33 & 0.933 & 2.256 \\
\hline$i_{1 m}{ }^{*}$ & 0.345 & 0.565 & 1.553 & 1.3 & 0.941 \\
\hline$j_{1 m}{ }^{*}$ & 0.992 & 1.268 & 1.677 & 1.754 & 1.423 \\
\hline$j_{2 m}{ }^{*}$ & 0.141 & 0.12 & 0.958 & 0.568 & 0.447 \\
\hline$V_{m}{ }^{*}$ & 0.449 & 0.516 & 1.592 & 1.257 & 0.965 \\
\hline$\eta^{*}$ & 0.537 & 0.727 & 1.403 & 1.573 & 1.061 \\
\hline$\theta_{1}{ }^{*}$ & 1.953 & 2.834 & 4.1 & 3.712 & 3.15 \\
\hline$\theta_{2}{ }^{*}$ & 0.053 & 0.037 & 0.86 & 0.34 & 0.323 \\
\hline$m_{\Sigma}{ }^{*}$ & 1.456 & 1.856 & 0.976 & 1.03 & 1.33 \\
\hline$K^{*}$ & 2.858 & 3.331 & 1.3 & 1.376 & 2.216 \\
\hline
\end{tabular}

Based on the results obtained, the following conclusions can be drawn. Inductor of LPIEC must have the maximum outer $D_{e x 1}=0.1 \mathrm{~m}$ and minimum inner $D_{i n 1}=0.01 \mathrm{~m}$ diameters. The armature should have the maximum outer diameter $D_{e x 2}=0.1 \mathrm{~m}$, and the 
thickness of its wire should be minimum $h_{z 2}=1 \mathrm{~mm}$. These parameters accept the limiting functional limitations (Table 1) and correspond to the parameters of LPIEC of basic design in all options of the effectiveness evaluation strategy.

The height of the inductor should be less than that of LPIEC of the basic design and, depending on the strategy options, averages $H_{1}=6.8 \mathrm{~mm}$. Here, the number of turns of the inductor in options I and II should be greater, and in options III and IV less than of the basic design of LPIEC, averaging $w_{1}=56$ turns of the wire with cross section $h_{z 1} \times h_{r 1}=1.5 \times 3.3 \mathrm{~mm}^{2}$. The armature of the optimized converter should be made with large inner diameter $D_{i n 2}=7 \mathrm{~mm}$ and significantly larger height $H_{2}=9.8 \mathrm{~mm}$, with large number of turns $w_{2}=140$ and wire width $h_{r 2}=2.6 \mathrm{~mm}$.

CES of optimal LPIEC should have less energy in options I and II, while its average value should be higher than that of LPIEC of basic design and be $W_{0}=312 \mathrm{~J}$. Voltage of CES in options I and II is low and averages $U_{0}=200 \mathrm{~V}$, and in options III and IV - the maximum $U_{0}=500 \mathrm{~V}$. The average values of maximum current densities as compared to LPIEC of the basic design in the inductor conductors are increased to $j_{1 m}=423 \mathrm{~A} / \mathrm{mm}^{2}$, and in the armature conductors are reduced to $j_{12 m}=342 \mathrm{~A} / \mathrm{mm}^{2}$.

Maximum speed and efficiency are reduced in strategy options I and II and increased in options III and IV. For example, in option III of the strategy, maximum speed is $V_{m}=13.4 \mathrm{~m} / \mathrm{s}$, and the efficiency in option IV is $\eta=16.2 \%$.

Compared to LPIEC of the basic design, in optimized converters, the temperature rise of the inductor increases, and the armature temperature rise decreases, averaging $\theta_{1}=1.17{ }^{\circ} \mathrm{C}$ and $\theta_{2}=0.31{ }^{\circ} \mathrm{C}$. The weight of copper wire increases on average to $m_{\Sigma}=1.14 \mathrm{~kg}$. Compared to the basic design of LPIEC, the integral efficiency criteria of optimized converters increase on average to a value $K^{*}=2.2$.

Fig. 1 shows the electromechanical characteristics of LPIEC of speed purpose, optimal in strategy option IV. A feature of these characteristics is that the maximum values of current densities in the windings of the inductor and the armature occur almost simultaneously, which causes the nature of the change in $\operatorname{EDF} f_{z}$. Movement of the armature with A begins in $0.2 \mathrm{~ms}$ after the start of the working process.

The results of the choice of parameters of LPIEC of power purpose. Let us consider four options of the strategy for evaluating the effectiveness of LPIEC for power purpose (Table 2). In option II, the amplitude $f_{z m}$ and the value of the impulse $F_{z}$ of EDF are estimated most highly and equally. In option III, the amplitude of EDU $f_{z m}$ is most evaluated. In option IV, the value of the impulse of $\operatorname{EDU} F_{z}$ is most evaluated.

Table 5, 6 show the average values of parameters and indicators of LPIEC for power purpose.

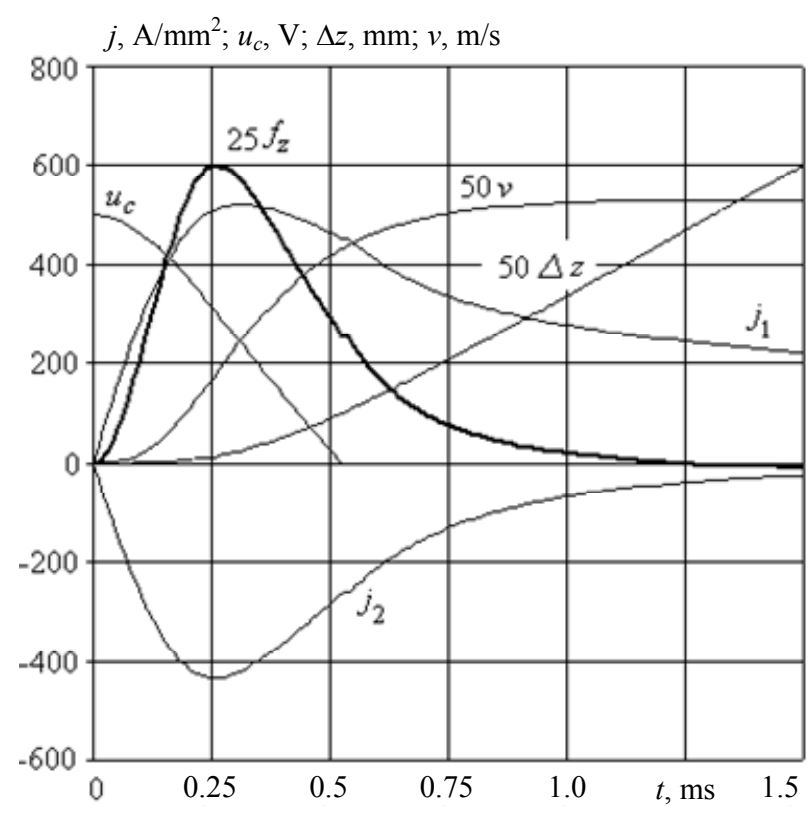

Fig. 1. Electromechanical characteristics of the optimal LPIEC of speed purpose (option of strategy IV)

Table 5

Relative parameters of LPIEC of power purpose

\begin{tabular}{|c|c|c|c|c|c|}
\hline \multirow[t]{2}{*}{ Parameter } & \multicolumn{4}{|c|}{$\begin{array}{c}\text { Strategy options for estimation of } \\
\text { LPIEC efficiency }\end{array}$} & \multirow{2}{*}{$\begin{array}{l}\text { Average } \\
\text { value }\end{array}$} \\
\hline & I & II & III & IV & \\
\hline$D_{e x 1}{ }^{*}$ & 1.0 & 1.0 & 1.0 & 1.0 & 1.0 \\
\hline$D_{i n 1}{ }^{*}$ & 1.1 & 1.0 & 1.0 & 1.0 & 1.0 \\
\hline$H_{1}{ }^{*}$ & 1.04 & 1.04 & 1.06 & 0.86 & 1.0 \\
\hline$w_{1}{ }^{*}$ & 0.87 & 1.39 & 1.09 & 0.91 & 1.07 \\
\hline$h_{z 1}{ }^{*}$ & 1.11 & 0.66 & 0.89 & 1.11 & 0.94 \\
\hline$h_{r 1}{ }^{*}$ & 1.04 & 1.04 & 1.06 & 0.88 & 1.01 \\
\hline$D_{e x 2}{ }^{*}$ & 1.0 & 1.0 & 1.0 & 1.0 & 1.0 \\
\hline$D_{i n 2}{ }^{*}$ & 1.2 & 1.2 & 1.3 & 1.4 & 1.275 \\
\hline $\mathrm{H}_{2}{ }^{*}$ & 5.0 & 5.0 & 6.0 & 3.84 & 4.96 \\
\hline$w_{2}{ }^{*}$ & 2.1 & 2.3 & 2.2 & 2.2 & 2.2 \\
\hline$K_{w 2}{ }^{*}$ & 2 & 2 & 2 & 2 & 2 \\
\hline$h_{z 2}{ }^{*}$ & 1.0 & 1.0 & 1.0 & 1.0 & 1.0 \\
\hline$h_{r 2}{ }^{*}$ & 2.5 & 2.5 & 2.9 & 1.92 & 2.46 \\
\hline
\end{tabular}

Table 6

Relative indicators of LPIEC of power purpose

\begin{tabular}{|c|c|c|c|c|c|}
\hline \multirow{2}{*}{ Indicator } & \multicolumn{4}{|c|}{ Strategy options for estimation of } & \multirow{2}{*}{\begin{tabular}{c}
\multirow{2}{*}{ Average } \\
value
\end{tabular}} \\
\cline { 2 - 5 } & I & II & III & IV & \\
\hline$W_{0}{ }^{*}$ & 0.625 & 2.08 & 1.875 & 2.08 & $\mathbf{1 . 6 6 5}$ \\
\hline$U_{0}{ }^{*}$ & 0.375 & 1.25 & 1.125 & 1.2 & $\mathbf{0 . 9 8 8}$ \\
\hline$C^{*}$ & 4.444 & 1.333 & 1.481 & 1.45 & $\mathbf{2 . 1 7 7}$ \\
\hline$i_{1 m}{ }^{*}$ & 0.818 & 0.993 & 1.234 & 1.616 & $\mathbf{1 . 1 6 5}$ \\
\hline$j_{1 m}{ }^{*}$ & 0.703 & 1.43 & 1.333 & 1.662 & $\mathbf{1 . 2 8 2}$ \\
\hline$j_{2 m}{ }^{*}$ & 0.146 & 0.288 & 0.243 & 0.428 & $\mathbf{0 . 2 7 6}$ \\
\hline$f_{z m}{ }^{*}$ & 0.382 & 1.47 & 2.018 & 1.361 & $\mathbf{1 . 3 0 8}$ \\
\hline$F_{z}{ }^{*}$ & 0.891 & 3.147 & 2.918 & 3.318 & $\mathbf{2 . 5 6 8}$ \\
\hline$\theta_{1}{ }^{*}$ & 1.062 & 3.382 & 3.181 & 4.198 & $\mathbf{2 . 9 5 6}$ \\
\hline$\theta_{2}{ }^{*}$ & 0.051 & 0.19 & 0.125 & 0.291 & $\mathbf{0 . 1 6 4}$ \\
\hline$m_{\Sigma}{ }^{*}$ & 1.832 & 1.832 & 2.032 & 1.456 & $\mathbf{1 . 7 8 8}$ \\
\hline$K^{*}$ & 2.664 & 2.147 & 2.159 & 2.047 & $\mathbf{2 . 2 5 4}$ \\
\hline
\end{tabular}


The height of the inductor of this converter should be almost the same as that of LPIEC of the basic design, and should be on average $H_{1}=10 \mathrm{~mm}$. At the same time, the number of inductor turns in strategy options I and IV should be less, and in strategy options II and III more than in the basic design of LPIEC, averaging $w_{1}=50$ turns. The cross section of the inductor wire $h_{z 1} \times h_{r 1}=1.7 \times 4.8 \mathrm{~mm}^{2}$ should be almost the same. The armature should be made with slightly larger inner diameter $D_{i n 2}=7.6 \mathrm{~mm}$ and significantly larger height $\mathrm{H}_{2}=12.4 \mathrm{~mm}$. This armature should have greater number of turns of wire $w_{2}=176$, which should be laid in 4 layers, and large wire width $h_{r 2}=3.0 \mathrm{~mm}$.

CES of LPIEC for power purpose should have less energy only in option I of strategy, in which all indicators are evaluated equally. The average value of the energy of CES should be higher than that of LPIEC of the basic design and be $W_{0}=400 \mathrm{~J}$. The voltage of CES in the option of strategy $\mathrm{I}$ is low and is $U_{0}=150 \mathrm{~V}$, and in other variants it is increased and is $U_{0}=450-500 \mathrm{~V}$. The capacitance of CES increases in all variants of the strategy, averaging $C=6530 \mu \mathrm{F}$.

The average values of the maximum current densities compared with LPIEC of the main design in inductor conductors are on average increased to $j_{1 m}=438 \mathrm{~A} / \mathrm{mm}^{2}$, and in armature conductors reduced to $j_{2 m}=246 \mathrm{~A} / \mathrm{mm}^{2}$. The amplitude and value of the impulse of EDF are increased except for option of strategy I. Thus, in the option of strategy III, the amplitude of EDF is $f_{z m}=40.7 \mathrm{kN}$, and in the option of strategy IV, the value of the impulse of EDF is $F_{z}=30.11 \mathrm{~N} \cdot \mathrm{s}$. In all options of the strategy, compared to LPIEC of the basic design, the temperature rise of the inductor increases, and the armature temperature rise decreases, averaging $\theta_{1}=1.18{ }^{\circ} \mathrm{C}$ and $\theta_{2}=0.24{ }^{\circ} \mathrm{C}$, respectively. The mass of copper wire increases on average to $m_{\Sigma}=1.54 \mathrm{~kg}$. Compared to the basic design of LPIEC, the integral efficiency criteria of optimized converters of power purpose increase on average to the value $K^{*}=2.25$.

Fig. 2 shows the electrodynamic characteristics of LPIEC for power purpose, optimal in strategy option IV. Compared with LPIEC speed purpose, in this converter the electrodynamic processes proceed with large delay in time, with large amplitudes of the current densities in the inductor and armature, and also EDF.

\section{Conclusions}

1. An optimization approach has been developed for choice parameters of LPIEC with a multi-turn shortcircuited armature, which consists in finding the maximum of the integral efficiency criterion that takes into account the maximum speed and efficiency of the converter of speed purpose, amplitude and value of the impulse of EDF of the converter of power purpose with minimum temperature rises, mass of active elements and current in inductor. The optimization uses a chain mathematical model that takes into account the interconnected electrical, magnetic, thermal, and mechanical processes of LPIEC.

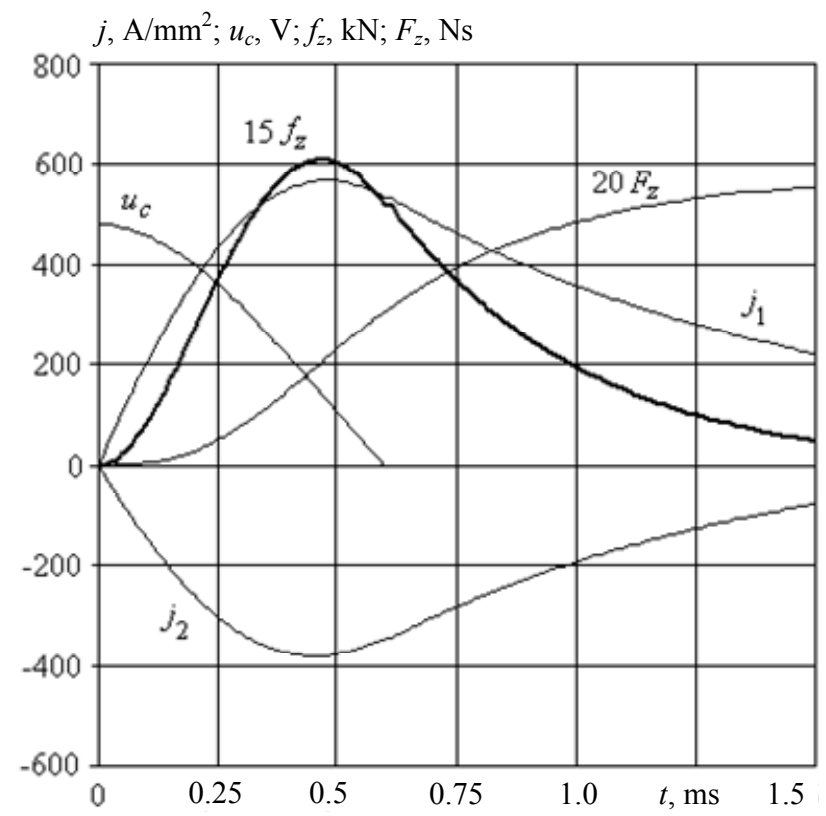

Fig. 2. Electrodynamic characteristics of the optimal LPIEC of power purpose (option of strategy IV)

2. A technique has been developed for finding the maximum of the integral criterion of LPIEC efficiency in the search space using a global optimization method that performs a random search for parameters, preventing it from falling into a local maximum, and a local method that provides a parameter region tightening with a global maximum to the minimum size. Genetic algorithms are used as a global optimization method, and the NelderMead method is used as a local optimization method.

3 . The values of the electrical parameters of the capacitive energy storage and the geometrical parameters of LPIEC are determined, which provide the highest values of the integral efficiency criterion depending on the adopted option of the effectiveness evaluation strategy. In optimized converters for speed and power purposes, the integral efficiency criteria are on average 2.2 times higher than in LPIEC of basic design.

\section{REFERENCES}

1. Balikci A., Zabar Z., Birenbaum L., Czarkowski D. Improved performance of linear induction launchers. IEEE Transactions on Magnetics, 2005, vol.41, no.1, pp. 171-175. doi: 10.1109/tmag.2004.839283.

2. Tomashevsky D.N., Koshkin A.N. Modeling of linear impulse electric motors. Russian Electrical Engineering, 2006, no.1, pp. 24-27. (Rus).

3. Chemerys V.T., Bolyukh V.F. Prospectives of new coilgun design development. Artillery and small arms, 2008, no.3, pp. 44-52.

4. D.-K. Lim, D.-K. Woo, I.-W. Kim, D.-K. Shin, J.-S. Ro, T.K. Chung, H.-K. Jung. Characteristic Analysis and Design of a Thomson Coil Actuator Using an Analytic Method and a Numerical Method. IEEE Transactions on Magnetics, 2013, vol.49, no.12, pp. 5749-5755. doi: 10.1109/tmag.2013.2272561. 5. Bolyukh V.F., Shchukin I.S. Lineinye induktsionnodinamicheskie preobrazovateli [Linear induction-dynamic converters]. Saarbrucken, Germany, LAP Lambert Academic Publ., 2014. 496 p. (Rus).

6. Bolyukh V.F., Oleksenko S.V., Shchukin I.S. Comparative analysis of linear pulse electromechanical converters 
electromagnetic and induction types. Technical Electrodynamics, 2016, no.5, pp. 46-48. (Rus).

7. Bissal A., Magnusson J., Engdahl G. Comparison of two ultra-fast actuator concept. IEEE Transactions on Magnetics, 2012, vol.48, no.11, pp. 3315-3318. doi: 10.1109/tmag.2012.2198447.

8. J. Young-woo, L. Hyun-wook, L. Seok-won. High-speed AC circuit breaker and high-speed OCD. Proceeding of the conf. «22-th international conference on electricity distribution». 2013, 10-13 June, Stockholm. - Paper 608.

9. Li W., Koh C.S. Parametric analysis of Thomson-coil actuator using adaptive equivalent circuit method. Digests of the 2010 14th Biennial IEEE Conference on Electromagnetic Field Computation, May 2010, pp. 1-9. doi: 10.1109/cefc.2010.5481673.

10. Bolyukh V.F., Kocherga A.I., Schukin I.S. Investigation of a linear pulse-induction electromechanical converter with different inductor power supply circuits. Electrical engineering \& electromechanics, 2018, no.1, pp. 21-28. (Rus). doi: 10.20998/2074-272X.2018.1.03.

11. Bolyukh V.F., Schukin I.S. Investigation of thermal processes in a linear pulse-induction electromechanical converter of cyclic action. Electrical engineering \& electromechanics, 2017, no.5, pp. 14-22. doi: 10.20998/2074272X.2017.5.02.

12. Nolan R., Pillay P., Haque T. Application of genetic algorithms to motor parameter determination. Proceedings of
1994 IEEE Industry Applications Society Annual Meeting, Baltimore, USA, 1994, pp. 42-54. doi: 10.1109/ias.1994.345500.

13. Bolyukh V.F., Lysenko L.I., Bolyukh E.G. Parameters of high-efficiency pulsed inductive electromechanical converters. Russian Electrical Engineering, 2004, vol.75, no.12, pp. 1-11.

14. Nelder J.A., Mead R. A Simplex Method for Function Minimization. The Computer Journal, 1965, vol.7, no.4, pp. 308-313. doi: 10.1093/comjnl/7.4.308.

Received 04.09.2018

V.F. Bolyukh ${ }^{1}$, Doctor of Technical Science, Professor, I.S. Schukin ${ }^{2}$, Candidate of Technical Science, Associate Professor,

${ }^{1}$ National Technical University «Kharkiv Polytechnic Institute», 2, Kyrpychova Str., Kharkiv, 61002, Ukraine, phone +380 577076427 ,

e-mail: vfbolyukh@gmail.com

${ }^{2}$ Firm Tetra, LTD,

2, Kyrpychova Str., Kharkiv, 61002, Ukraine, phone +380 577076427 ,

e-mail: tech@tetra.kharkiv.com.ua

How to cite this article:

Bolyukh V.F., Schukin I.S. An optimization approach to the choice of parameters of linear pulse induction electromechanical converter. Electrical engineering \& electromechanics, 2018, no.6, pp. 18-25. doi: 10.20998/2074272X.2018.6.02. 